\title{
The Effects of Allopurinol on the Carotid Intima-media Thickness in Patients with Type 2 Diabetes and Asymptomatic Hyperuricemia: A Three-year Randomized Parallel-controlled Study
}

\author{
Peng Liu ${ }^{1,2}$, Huayi Wang ${ }^{3}$, Fengmei Zhang ${ }^{2}$, Yingying Chen ${ }^{4}$, \\ Debao Wang ${ }^{2}$ and Yangang Wang ${ }^{1}$
}

\begin{abstract}
Objective The aim of this study was to investigate the long-term effective control of serum uric acid by allopurinol on the carotid intima-media thickness (IMT) in patients with type 2 diabetes (T2DM) and asymptomatic hyperuricemia (HUA).

Methods This was a randomized open parallel-controlled study. In this study, 176 patients with T2DM and asymptomatic HUA were randomly allocated to the conventional or allopurinol treatment groups on the basis of a computer-generated random number table. Changes in the carotid IMT, biochemical indexes, high sensitive C-reactive protein (hs-CRP) and the incidence of hypertension in patients before and after three years of treatment were examined and compared between the groups.

Results There were no statistically significant differences in the baseline characteristics of the study participants between the two treatment groups ( $p>0.05$ for all). Nevertheless, the serum uric acid, triglyceride, and hs-CRP levels and the homeostasis assessment for insulin resistance (HOMA-IR), systolic blood pressure, diastolic blood pressure and the carotid IMT in the allopurinol group were significantly lower than those in the conventional group after three years of treatment $(\mathrm{p}<0.01$ for all). The intention-to-treat analysis indicated that the incidence of new-onset hypertension in the allopurinol group showed a declining trend compared to that in the conventional treatment group $(6.8 \%$ vs. $13.6 \%, \mathrm{p}>0.05)$.

Conclusion The long-term effective control of serum uric acid by allopurinol may improve insulin resistance, decrease the serum levels of hs-CRP, reduce the carotid IMT, and may delay the development of atherosclerosis in patients with T2DM and asymptomatic HUA.
\end{abstract}

Key words: hyperuricemia, type 2 diabetes, high sensitive C-reactive protein, carotid intima-media thickness

(Intern Med 54: 2129-2137, 2015)

(DOI: 10.2169/internalmedicine.54.4310)

\section{Introduction}

Hyperuricemia (HUA) is defined as a serum uric acid level $>420 \mu \mathrm{mol} / \mathrm{L}$ in men and $>360 \mu \mathrm{mol} / \mathrm{L}$ in women. HUA without the onset of gout is classified as asymptomatic HUA (1). Epidemiological studies have indicated that HUA is relatively prevalent in the general population. The preva- lence rate of HUA in the coastal areas of Shandong reached $16.7 \%$ in a 2009 survey, approaching the levels of Western developed countries (2). HUA is closely related to gouty arthritis, metabolic syndrome, hypertension, cardiovascular diseases and chronic kidney diseases and is an independent risk factor for these diseases (3). Furthermore, HUA is significantly associated with type 2 diabetes (T2DM), and the risk of T2DM gradually increases with elevating serum uric

\footnotetext{
${ }^{1}$ Department of Endocrinology, The Affiliated Hospital of Qingdao University, China, ${ }^{2}$ Department of Endocrinology, Laiwu Hospital Affiliated with Taishan Medical College, China, ${ }^{3}$ Laiwu Municipal Center for Disease Control and Prevention, China and ${ }^{4}$ Department of Clinical Laboratory, Laiwu Hospital Affiliated with Taishan Medical College, China

Received for publication October 20, 2014; Accepted for publication January 13, 2015

Correspondence to Dr. Yangang Wang, ygwang1966@126.com
} 
acid levels $(4,5)$. Previous studies have identified many risk factors for atherosclerosis, such as hyperglycemia, hypertension, dyslipidemia, and smoking. However, intensive multifactorial therapy by a strict control of blood glucose, blood pressure and the regulation of blood lipids cannot effectively prevent the development of atherosclerosis, residual macrovascular risk remains (6). Several studies have shown that effective control of serum uric acid can significantly reduce cardiovascular events. In the Losartan Intervention for Endpoint Reduction (LIFE) study, Dahlof et al. (7) showed that $29 \%$ of the cardio-protective effect of losartan was attributed to this drug's hypouricemic properties. A recent study (8) indicated that high-dose allopurinol can cause regression of left ventricular mass through the strict control of blood uric acid levels; therefore allopurinol may be useful for reducing cardiovascular events in T2DM patients with left ventricular hypertrophy. Several recent studies have found that asymptomatic HUA is an important risk factor for the occurrence and progression of atherosclerosis in patients with T2DM $(9,10)$. The carotid intima-media thickness (IMT) is an early marker of atherosclerosis and closely related to macrovascular diseases. Currently, there is a lack of research on whether the long-term effective control of serum uric acid can delay the progression of carotid atherosclerosis in patients with T2DM and asymptomatic HUA. In this study, a randomized parallel-controlled trial was performed to investigate the effects of allopurinol treatment on the carotid IMT and the levels of serum high sensitive Creactive protein (hs-CRP) to explore the possible therapeutic mechanism of the long-term effective control of serum uric acid on atherosclerosis.

\section{Materials and Methods}

\section{Patients}

The clinical trial included 176 patients with T2DM and asymptomatic HUA receiving inpatient or outpatient therapies from October 2009 in the Endocrinology Department of Laiwu Hospital affiliated with Taishan Medical College. There were 85 males and 91 females, with an average age of 51 years. The inclusion criteria were as follows: 1) patients that met the 1999 World Health Organization diagnostic criteria for T2DM and the asymptomatic HUA diagnostic criteria; 2) an age $<70$ years; 3) good glycemic control and a stable illness condition; 4) a urinary albumin excretion rate (UAER) <20 $\mu \mathrm{g} / \mathrm{min}$; 5) serum uric acid levels between 420 and $476 \mu \mathrm{mol} / \mathrm{L}$ after one month on a low-purine diet; 6) no administration of medications affecting uric acid metabolism over the last three months, such as thiazide diuretics, compound reserpine, pyrazinamide, nifedipine, propranolol, allopurinol, benzbromarone; 7) no diseases which affect uric acid metabolism, such as chronic renal insufficiency; and 8) no administration of angiotensin-converting enzyme inhibitors, angiotensin receptor blockers, uric acid synthesisinhibiting drugs, uricosuric drugs or lipid lowering drugs for approximately three months. The exclusion criteria were as follows: previous history of gout, primary renal diseases, malnutrition and dehydration, hypertension, severe dyslipidemia, acute metabolic disorders in diabetes, malignancy, or severe cardiac, hepatic, or cerebral diseases. The termination criteria were as follows: the serum uric acid level gradually increased to more than $476 \mathrm{umol} / \mathrm{L}$ (1); an acute attack of gout; or a serious adverse drug reaction that patients were unable to tolerate.

From the perspectives of effectiveness and statistics, we chose $\alpha=0.05$ and $\beta=0.2$ (i.e., power $=80 \%$ ) as the criteria for the sample size calculation. According to the corresponding reference, the standard deviation of the carotid IMT in patients with T2DM and HUA is $0.29 \mathrm{~mm}$ (10). The effective percentage in the carotid IMT of the allopurinol group was expected to be $15 \%$ higher than that of the conventional therapy group. The calculated results showed that a minimum of 66 cases were required for each group, with an estimated loss rate of approximately $20 \%$. Considering that the serum uric acid level gradually increased with changing renal function, a total of 200 cases were included in this study.

All selected patients were randomly allocated to the conventional or allopurinol treatment group by a computergenerated random number table (Fig. 1). The conventional group included 88 patients, with 14 who had serum uric acid levels which gradually became $>476$ umol/L (excluded), two with acute gout (excluded), and two who were loss to follow-up. Ultimately, the conventional group included 70 patients (32 males and 38 females with an average age of $51 \pm 11$ years) and a course of diabetes mellitus for $4.9 \pm 1.7$ years. The allopurinol group had 88 patients, including one case of severe vomiting (excluded), two with liver damage (excluded), and three who were loss to followup. Ultimately, the allopurinol group included 82 patients (38 males and 44 females, with an average age of $50 \pm 10$ years) and a course of diabetes mellitus for $5.1 \pm 2.0$ years. This research was approved by the ethics committee of the hospital, and informed consent was provided by all patients.

\section{Determination of evaluation indicators}

Both groups of patients received diabetes education with diet control, low-purine diet, and adequate exercise. For the majority of the patients, oral hypoglycemic drugs combined with insulin therapy were used for blood glucose control; for a small number of patients, oral hypoglycemic drugs were administered to control the blood glucose levels within the target range. To treat abnormal blood lipid levels, statins were routinely used when the total cholesterol (TC) level was greater than $4.5 \mathrm{mmol} / \mathrm{L}$ and/or the low-density lipoprotein cholesterol (LDL-C) was greater than $2.6 \mathrm{mmol} / \mathrm{L}$. The aspirin regimen followed the 2007 and 2010 editions of the Chinese Guideline of Type 2 Diabetes Prevention and Treatment. The two groups of patients had no statistically significant differences before and after the treatment in regards to the enhanced glucose control, blood lipid regulation, and as- 


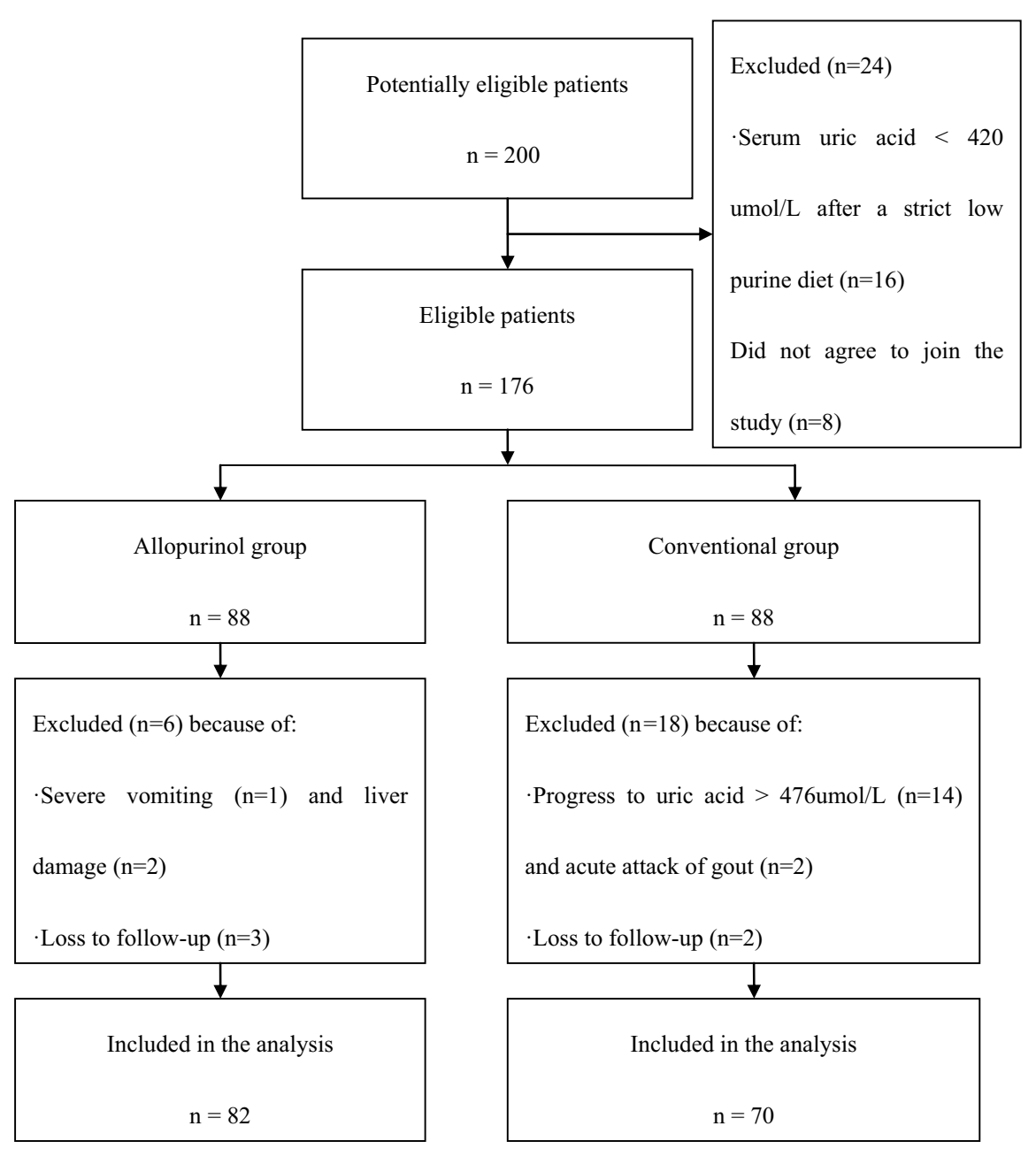

Figure 1. Study design and inclusion criteria.

pirin regimen ( $>0.05$, Table 1). The patients in the allopurinol group were given allopurinol (starting from $100 \mathrm{mg} /$ day) when the blood uric acid level was greater than $420 \mu \mathrm{mol} / \mathrm{L}$. These patients were re-examined monthly to test the blood uric acid level, and the dose of allopurinol was adjusted to maintain a serum uric acid level less than $360 \mu \mathrm{mol} / \mathrm{L}$. The patients in the conventional therapy group received no uric acid lowering therapy when the blood uric acid level was less than $476 \mu \mathrm{mol} / \mathrm{L}$. The patients with blood uric acid levels exceeding $476 \mu \mathrm{mol} / \mathrm{L}$ were excluded from the following examination.

During the study, the patients received telephone and outpatient follow-ups. A healthy lifestyle (e.g., at least three 30-minute sessions of aerobic exercise per week; no drinking and no smoking) was recommended by group counseling supplemented with audiovisual and printed materials monthly. All patients went on stringent low purine diet and stopped drinking. The fasting plasma glucose (FPG) and postprandial plasma glucose ( $2 \mathrm{hPG}$ ) levels were regularly determined, and the glycosylated hemoglobin A1c (HbAlc) level was checked every 2-3 months. If the FPG level was $>7.2 \mathrm{mmol} / \mathrm{L}$ and the $2 \mathrm{hPG}$ level was $>10 \mathrm{mmol} / \mathrm{L}$, the dose of insulin (basal insulin or premixed combined human insulin analogues) and/or the dose of oral hypoglycemic drugs (metformin, glimepiride or pioglitazone) were adjusted to maintain the blood glucose level in the target range. Acarbose and/or prandial insulin analogues were added if the $2 \mathrm{hPG}$ level remained $>10 \mathrm{mmol} / \mathrm{L}$ after any type of hypoglycemic administration. The blood glucose target level was a FPG level $<7.2 \mathrm{mmol} / \mathrm{L}$ and a $2 \mathrm{hPG}$ level of 7.8-11.1 $\mathrm{mmol} / \mathrm{L}$. Blood lipids were detected every three months. Statins were routinely used if TC $>4.5 \mathrm{mmol} / \mathrm{L}$ and/or LDL$\mathrm{C}>2.6 \mathrm{mmol} / \mathrm{L}$. The blood pressure was monitored monthly. An appropriately sized cuff (cuff bladder encircling at least $80 \%$ of the arm) was used to ensure accuracy. Two measurements were made and the average was recorded. To prevent any observer bias at the time of measuring blood pressure (BP), the nurse in charge was prevented from knowing which patients were under treatment of allopurinol. The patients primarily received a calcium antagonist when the blood pressure exceeded 140/90 mmHg. If unsuccessful, an angiotensin-converting enzyme inhibitor or angiotensin II receptor blockers were added as a supplemental therapy. The blood pressure target was $130 / 80 \mathrm{mmHg}$. Atorvastatin and losartan were not selected due to the impact of these drugs on the level of serum uric acid. The carotid IMT and hsCRP level were monitored every six months. The total course of treatment was three years. 
Table 1. Baseline Characteristics of the Study Participants.

\begin{tabular}{|c|c|c|c|c|c|c|}
\hline & $\begin{array}{l}\text { Allopurinol } \\
\text { group }(n=88)\end{array}$ & $\begin{array}{l}\text { conventional } \\
\text { group }(n=88)\end{array}$ & $\mathrm{p}$ value & $\begin{array}{l}\text { Allopurinol } \\
\text { group } \\
(\mathrm{n}=82)\end{array}$ & $\begin{array}{l}\text { conventional } \\
\text { group } \\
(\mathrm{n}=70)\end{array}$ & $\mathrm{p}$ value \\
\hline Age, yrs & $50 \pm 10$ & $51 \pm 11$ & 0.553 & $50 \pm 10$ & $51 \pm 11$ & 0.747 \\
\hline Gender, \% (male) & $42(47.7 \%)$ & $43(48.9 \%)$ & 0.880 & $38(46.3 \%)$ & $32(45.7 \%)$ & 0.938 \\
\hline Diabetes duration, yrs & $5.0 \pm 2.0$ & $5.0 \pm 1.8$ & 0.919 & $5.1 \pm 2.0$ & $4.9 \pm 1.7$ & 0.566 \\
\hline Body mass index, $\mathrm{kg} / \mathrm{m}^{2}$ & $25.0 \pm 1.1$ & $25.2 \pm 1.1$ & 0.226 & $25.0 \pm 1.2$ & $25.1 \pm 1.1$ & 0.640 \\
\hline $\begin{array}{l}\text { Systolic blood pressure, } \\
\mathrm{mmHg}\end{array}$ & $121 \pm 8$ & $123 \pm 8$ & 0.451 & $121 \pm 8$ & $121 \pm 8$ & 0.872 \\
\hline $\begin{array}{l}\text { Diastolic blood pressure, } \\
\mathrm{mmHg}\end{array}$ & $74 \pm 6$ & $75 \pm 7$ & 0.379 & $74 \pm 6$ & $74 \pm 7$ & 0.948 \\
\hline $\begin{array}{l}\text { Fasting plasma glucose, } \\
\mathrm{mmol} / \mathrm{L}\end{array}$ & $6.25 \pm 0.58$ & $6.24 \pm 0.55$ & 0.954 & $6.22 \pm 0.57$ & $6.21 \pm 0.56$ & 0.906 \\
\hline $\begin{array}{l}\text { 2h postprandial plasma } \\
\text { glucose, } \mathrm{mmol} / \mathrm{L}\end{array}$ & $9.82 \pm 0.78$ & $9.85 \pm 0.76$ & 0.743 & $9.81 \pm 0.78$ & $9.83 \pm 0.78$ & 0.861 \\
\hline $\mathrm{HbA} 1 \mathrm{c}, \%$ & $6.89 \pm 0.59$ & $6.91 \pm 0.64$ & 0.845 & $6.9 \pm 0.5$ & $6.9 \pm 0.6$ & 0.793 \\
\hline FINS, mU/L & $12.02 \pm 2.65$ & $12.13 \pm 2.23$ & 0.774 & $11.99 \pm 2.64$ & $11.94 \pm 2.15$ & 0.908 \\
\hline HOMA-IR & $3.42 \pm 1.05$ & $3.46 \pm 0.75$ & 0.754 & $3.39 \pm 1.05$ & $3.36 \pm 0.73$ & 0.839 \\
\hline HOMA- $\beta$ & $4.49 \pm 0.35$ & $4.51 \pm 0.31$ & 0.827 & $4.48 \pm 0.35$ & $4.49_{ \pm} 0.30$ & 0.878 \\
\hline Total cholesterol, $\mathrm{mmol} / \mathrm{L}$ & $5.10 \pm 0.76$ & $5.12 \pm 0.73$ & 0.874 & $5.08 \pm 0.75$ & $5.06 \pm 0.76$ & 0.888 \\
\hline LDL-C, $\mathrm{mmol} / \mathrm{L}$ & $3.01 \pm 0.63$ & $2.99_{ \pm} 0.55$ & 0.743 & $2.98 \pm 0.64$ & $2.94 \pm 0.56$ & 0.695 \\
\hline $\mathrm{HDL}-\mathrm{C}, \mathrm{mmol} / \mathrm{L}$ & $1.06 \pm 0.18$ & $1.07 \pm 0.14$ & 0.735 & $1.06 \pm 0.17$ & $1.07 \pm 0.14$ & 0.812 \\
\hline Triglyceride, $\mathrm{mmol} / \mathrm{L}$ & $2.03 \pm 0.53$ & $2.00 \pm 0.43$ & 0.671 & $1.98 \pm 0.52$ & $1.94 \pm 0.43$ & 0.598 \\
\hline Serum uric acid, umol/L & $433 \pm 11$ & $434 \pm 10$ & 0.696 & $433 \pm 11$ & $432 \pm 9$ & 0.622 \\
\hline Serum creatinine, umol/L & $76.1 \pm 11.0$ & $76.2 \pm 10.8$ & 0.923 & $75.6 \pm 11.0$ & $75.0 \pm 10.9$ & 0.734 \\
\hline $\mathrm{GFR}, \mathrm{mL} / \mathrm{min} / 1.73 \mathrm{~m}^{2}$ & $91.3 \pm 18.2$ & $92.4 \pm 18.7$ & 0.765 & $90.1 \pm 17.5$ & $90.1 \pm 18.4$ & 0.992 \\
\hline hs-CRP, mg/L & $2.56 \pm 0.66$ & $2.63 \pm 0.64$ & 0.500 & $2.53 \pm 0.66$ & $2.48 \pm 0.59$ & 0.638 \\
\hline carotid IMT, mm & $1.07 \pm 0.22$ & $1.08 \pm 0.20$ & 0.791 & $1.06 \pm 0.22$ & $1.05 \pm 0.20$ & 0.720 \\
\hline Smoking, $\%$ & $32(36.4 \%)$ & $31(35.2 \%)$ & 0.875 & $29(35.4 \%)$ & $23(32.9 \%)$ & 0.745 \\
\hline Drug use & & & & & & \\
\hline Oral hypoglycemic drugs, $\%$ & $40(45.5 \%)$ & $38(43.2 \%)$ & 0.762 & $38(46.3 \%)$ & $30(42.9 \%)$ & 0.667 \\
\hline $\begin{array}{l}\text { Insulin combined with oral } \\
\text { hypoglycemic drugs, } \%\end{array}$ & $48(54.5 \%)$ & $50(56.8 \%)$ & 0.762 & $44(53.7 \%)$ & $40(57.1 \%)$ & 0.667 \\
\hline Lipid lowering drugs, $\%$ & $38(43.2 \%)$ & $41(46.6 \%)$ & 0.649 & $37(45.1 \%)$ & $30(42.9 \%)$ & 0.779 \\
\hline Antihypertensivedrugs, $\%$ & $0(0 \%)$ & $0(0 \%)$ & 1.000 & $0(0 \%)$ & $0(0 \%)$ & 1.000 \\
\hline Aspirin, \% & $43(48.9 \%)$ & $45(51.1 \%)$ & 0.763 & $41(50.0 \%)$ & $33(47.1 \%)$ & 0.725 \\
\hline
\end{tabular}

The primary outcome measured were changes in the carotid IMT between the two groups before and after treatment. The secondary endpoints were changes in the blood pressure, blood lipids, insulin resistance and serum levels of hs-CRP between the two groups before and after three years of treatment and changes in the incidence of hypertension in the two groups after treatment.

Before and after treatment, all patients underwent an oral glucose tolerance test and measurements of the height, weight, and blood pressure. FPG (measured after a 10 hours overnight fast) and $2 \mathrm{hPG}$ were assessed by the glucose oxidase method. HbA1c was detected by high-pressure liquid chromatography (Bio-Rad Laboratories, Hercules, USA). The serum creatinine, uric acid and lipid profiles, including triglyceride (TG), TC high-density lipoprotein cholesterol (HDL-C) and LDL-C, were determined by standard enzymatic procedures on an automated bioanalyzer (7600-020, Hitachi, Tokyo, Japan). The serum hs-CRP was measured by a particle-enhanced immunonephelometry assay (Beckman Coulter, Kraemer Blvd. Brea, USA). The fasting serum insulin (FINS) was assayed by a radioimmunoassay (Linco Research, St. Charles, USA). The above detected indices were measured by an experienced technician who was blinded to the study. Insulin resistance was indicated with an insulin resistance index for homeostasis model assessment (HOMA$\mathrm{IR}=\mathrm{FPG} \times$ FINS / 22.5). The pancreatic $\beta$ cell function was indicated by the $\beta$ cell function index for homeostasis model assessment [HOMA- $\beta=20 \times$ FINS / (FPG - 3.5)]. The glomerular filtration rate (GFR) was calculated by the Chronic Kidney Disease Epidemiology Collaboration (CKDEPI) formula $\left[\right.$ GFR $=\mathrm{a} \times(\text { serum creatinine } / \mathrm{b})^{\mathrm{c}} \times$ $\left.(0.993)^{\text {age }}\right]$ (11). The carotid IMT was detected using a highresolution ultrasound Doppler system (iU22, Philips ultrasound, Bothell, USA) with a 7.5-10.0 MHz liner array transducer. The patients were supine in the bed, with the head turned $45^{\circ}$ away from the examined side during the examination. The right and left common carotid arteries were scanned from proximally to distally, up to the point at which they bifurcate. Carotid IMT measurements were acquired at the far wall of the right and left common carotid arteries, approximately $1 \mathrm{~cm}$ proximal to the carotid bulb. The average value of the maximal thickness of each carotid artery was calculated to generate the carotid IMT. The intraobserver and inter-observer variability of the carotid IMT 

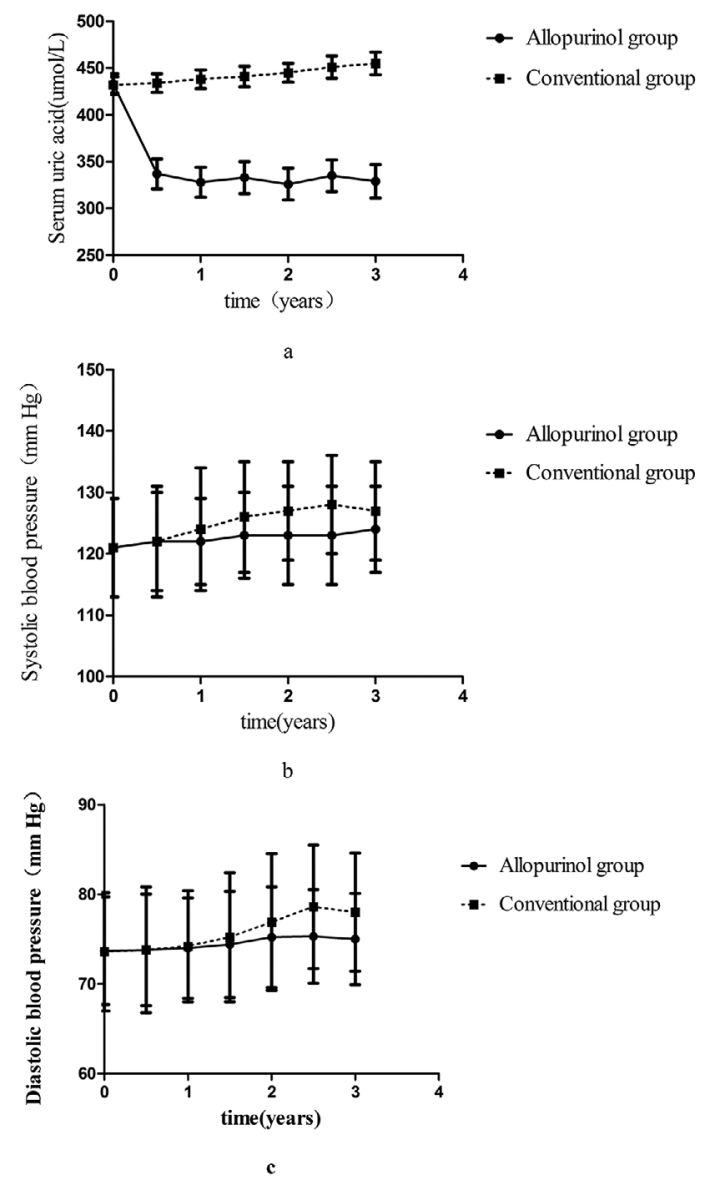

Figure 2. The interaction of time and group on the (a) serum uric acid level ( $F=379.862$, $p<0.001)$, (b) systolic blood pressure $(\mathrm{F}=\mathbf{2 . 1 9 4}, \mathrm{p}=\mathbf{0 . 0 4 1})$ and $(\mathrm{c})$ diastolic blood pressure $(\mathrm{F}=\mathbf{1 . 8 3 6}$, $\mathrm{p}=\mathbf{0 . 0 8 9}$ ).

measurements were less than $5 \%$. All scans were conducted by a designated investigator who was blinded to the clinical characteristics of the subjects.

\section{Statistical analysis}

Data analysis was performed using the SPSS 16.0 statistical software package (SPSS Inc., Chicago, USA). The measurement data were analyzed by the Shapiro-Wilk test, and the normal distribution data were expressed as the means \pm standard deviation (SD). HOMA- $\beta$ was a non-normally distributed variable and was analyzed after being converted to a normal distribution variable using the natural logarithm. The intra-group comparison of the pre- and post-treatment data was performed using the paired $t$ test. The inter-group comparison was performed using the $t$-test for independent samples. The enumeration data were analyzed by the chi square test. A general linear model was used to analyze the interactions of time and group during the study. The intention-totreat (ITT) analysis and per-protocol treated (PP) analysis were used to compare the incidence of hypertension between the two groups. A p value $<0.05$ was considered to be statistically significant.

\section{Results \\ Inter-group comparison of baseline characteristics}

There were no statistically significant differences regarding the age, gender, and course of disease between the two treatment groups ( $p>0.05$ for all). In addition, there were also no statistically significant differences in the blood pressure, glycemic control, blood lipid, uric acid, hs-CRP and carotid IMT between the two treatment groups $(\mathrm{p}>0.05$ for all) (Table 1).

\section{Intra-group comparison of evaluation indicators be- fore and after treatment}

After 3 years of conventional treatment, the levels of TG $[(2.23 \pm 0.43$ vs. $1.94 \pm 0.43) \mathrm{mmol} / \mathrm{L}, \quad \mathrm{p}<0.01], \quad$ hs-CRP [(2.83 \pm 0.61 vs. $2.48 \pm 0.59) \mathrm{mg} / \mathrm{L}, \mathrm{p}<0.01]$, systolic blood pressure $[(127 \pm 8$ vs. $121 \pm 8) \mathrm{mmHg}, \mathrm{p}<0.05]$ and diastolic blood pressure $[(78 \pm 7$ vs. $74 \pm 7) \mathrm{mmHg}, \mathrm{p}<0.05]$ increased significantly compared to pre-treatment levels, whereas TC [(4.16 \pm 0.27 vs. $5.06 \pm 0.76) \mathrm{mmol} / \mathrm{L}, \mathrm{p}<0.05]$ and $\mathrm{LDL}-\mathrm{C}$ $[(2.25 \pm 0.24$ vs. $2.94 \pm 0.56) \mathrm{mmol} / \mathrm{L}, \mathrm{p}<0.05]$ decreased significantly compared to pre-treatment levels. Although other indicators showed no significant changes ( $>0.05$ for all), there were clear increasing trends in the uric acid and carotid IMT levels compared to the pre-treatment levels. In the allopurinol group, there were significant declines in the uric acid levels [(329 \pm 18 vs. $433 \pm 11)$ umol/L, p<0.05], TC [(4.17 \pm 0.22 vs. $5.08 \pm 0.75) \mathrm{mmol} / \mathrm{L}, \mathrm{p}<0.05]$ and LDL-C levels $[(2.27 \pm 0.25$ vs. $2.98 \pm 0.64) \mathrm{mmol} / \mathrm{L}, \mathrm{p}<0.05]$ after 3 years of treatment, whereas the other indicators showed no significant changes ( $p>0.05$ for all). The minimum dose of allopurinol was $100 \mathrm{mg} /$ day, the maximum dose of allopurinol was $450 \mathrm{mg} / \mathrm{day}$, and the final average dose of allopurinol were $234 \pm 87 \mathrm{mg} /$ day.

\section{Inter-group comparison of evaluation indicators af- ter treatment}

After 3 years of treatment, the levels of uric acid, systolic blood pressure and diastolic blood pressure were more significantly reduced in the allopurinol group than in the conventional group. There was a significant interaction of time and group in the general linear model analysis in the serum uric acid level and systolic blood pressure, while the interaction of time and group was of borderline significance for diastolic blood pressure (Fig. 2). Further comparison of differences in the indicators between the pre- and posttreatment levels confirmed that the allopurinol treatment was more effective in reducing the uric acid level, TG, HOMAIR, systolic blood pressure, diastolic blood pressure, serum creatinine, hs-CRP, and carotid IMT and more effective in increasing GFR than the conventional treatment (Table 2). Moreover, allopurinol therapy was more efficacious in reducing the uric acid level, systolic blood pressure, hs-CRP and carotid IMT than conventional therapy in the patients 
Table 2. Post-treatment Changes of Indices between the Two Groups.

\begin{tabular}{|c|c|c|c|c|c|c|}
\hline & \multicolumn{2}{|c|}{ Post-treatment (mean \pm SD) } & \multirow[b]{2}{*}{$\mathrm{p}$ value } & \multicolumn{2}{|c|}{ Changing values } & \multirow[b]{2}{*}{$\mathrm{p}$ value } \\
\hline & $\begin{array}{l}\text { Allopurinol } \\
\text { group }(n=82)\end{array}$ & $\begin{array}{l}\text { conventional } \\
\text { group }(\mathrm{n}=70)\end{array}$ & & $\begin{array}{l}\text { Allopurinol } \\
\text { group }(\mathrm{n}=82)\end{array}$ & $\begin{array}{l}\text { conventional } \\
\text { group }(\mathrm{n}=70)\end{array}$ & \\
\hline Body mass index, $\mathrm{kg} / \mathrm{m}^{2}$ & $25.3 \pm 1.1$ & $25.4 \pm 1.0$ & 0.436 & $0.3 \pm 0.2$ & $0.3 \pm 0.2$ & 0.088 \\
\hline $\begin{array}{l}\text { Systolic blood pressure, } \\
\mathrm{mmHg}\end{array}$ & $124 \pm 7^{*}$ & $127 \pm 8$ & 0.014 & $2 \pm 3^{\dagger}$ & $6 \pm 2$ & $<0.001$ \\
\hline $\begin{array}{l}\text { Diastolic blood pressure, } \\
\mathrm{mmHg}\end{array}$ & $75 \pm 5^{\dagger}$ & $78 \pm 7$ & 0.003 & $1 \pm 3^{\dagger}$ & $4 \pm 2$ & $<0.001$ \\
\hline $\begin{array}{l}\text { Fasting plasma glucose, } \\
\mathrm{mmol} / \mathrm{L}\end{array}$ & $6.24 \pm 0.62$ & $6.22 \pm 0.52$ & 0.792 & $0.02 \pm 0.45$ & $0.01 \pm 0.28$ & 0.893 \\
\hline $\begin{array}{l}2 \mathrm{~h} \text { postprandial plasma } \\
\text { glucose } \mathrm{mmol} / \mathrm{L}\end{array}$ & $9.80 \pm 0.64$ & $9.84 \pm 0.61$ & 0.674 & $-0.01 \pm 1.05$ & $0.01 \pm 0.84$ & 0.894 \\
\hline $\mathrm{HbAlc}, \%$ & $7.0 \pm 0.5$ & $6.9 \pm 0.5$ & 0.775 & $0.1 \pm 0.7$ & $0.0 \pm 0.3$ & 0.586 \\
\hline FINS, mU/L & $12.36 \pm 2.76$ & $12.82 \pm 2.35$ & 0.284 & $0.37 \pm 0.69^{\dagger}$ & $0.88 \pm 0.67$ & $<0.001$ \\
\hline HOMA-IR & $3.49 \pm 1.01$ & $3.57 \pm 0.72$ & 0.584 & $0.10_{ \pm} 0.15^{\dagger}$ & $0.21 \pm 0.18$ & $<0.001$ \\
\hline НОМА- $\beta$ & $4.55 \pm 0.36$ & $4.51 \pm 0.30$ & 0.407 & $0.07 \pm 0.22$ & $0.02 \pm 0.25$ & 0.367 \\
\hline Total cholesterol, $\mathrm{mmol} / \mathrm{L}$ & $4.17 \pm 0.22$ & $4.16 \pm 0.27$ & 0.828 & $-0.91 \pm 0.78$ & $-0.90 \pm 0.79$ & 0.946 \\
\hline $\mathrm{LDL}-\mathrm{C}, \mathrm{mmol} / \mathrm{L}$ & $2.27 \pm 0.25$ & $2.25 \pm 0.24$ & 0.703 & $-0.71 \pm 0.67$ & $-0.69 \pm 0.54$ & 0.816 \\
\hline HDL-C, $\mathrm{mmol} / \mathrm{L}$ & $1.04 \pm 0.17$ & $1.03 \pm 0.13$ & 0.666 & $-0.02 \pm 0.03^{\dagger}$ & $-0.04 \pm 0.03$ & 0.001 \\
\hline Triglyceride, $\mathrm{mmol} / \mathrm{L}$ & $2.10 \pm 0.47$ & $2.23 \pm 0.43$ & 0.073 & $0.12 \pm 0.12^{\dagger}$ & $0.29 \pm 0.16$ & $<0.001$ \\
\hline Serum uric acid, umol/L & $329 \pm 18^{\dagger}$ & $455 \pm 12$ & $<0.001$ & $-104 \pm 19^{\dagger}$ & $23 \pm 11$ & $<0.001$ \\
\hline Serum creatinine, umol/L & $74.8 \pm 11.5$ & $78.4 \pm 12.8$ & 0.075 & $-0.8 \pm 3.3^{\dagger}$ & $3.4 \pm 5.4$ & $<0.001$ \\
\hline $\mathrm{GFR}, \mathrm{mL} / \mathrm{min} / 1.73 \mathrm{~m}^{2}$ & $89.3 \pm 17.8$ & $85.2 \pm 18.8$ & 0.178 & $-0.8 \pm 3.9^{\dagger}$ & $-4.9 \pm 5.0$ & $<0.001$ \\
\hline hs-CRP, mg/L & $2.68 \pm 0.67$ & $2.83 \pm 0.61$ & 0.146 & $0.15_{ \pm} 0.19^{\dagger}$ & $0.35 \pm 0.18$ & $<0.001$ \\
\hline carotid IMT, mm & $1.08 \pm 0.21$ & $1.12 \pm 0.21$ & 0.396 & $0.02 \pm 0.03^{\dagger}$ & $0.07 \pm 0.03$ & $<0.001$ \\
\hline Smoking, \% & $14(17.1 \%)$ & $10(14.3 \%)$ & 0.639 & $-15(-18.3 \%)$ & $-13(-18.6 \%)$ & 0.965 \\
\hline Drug use & & & & & & \\
\hline Oral hypoglycemic drugs, $\%$ & $25(30.5 \%)$ & $17(24.3 \%)$ & 0.394 & $-13(-15.9 \%)$ & $-13(-18.6 \%)$ & 0.657 \\
\hline $\begin{array}{l}\text { Insulin combined with oral } \\
\text { hypoglycemic drugs, } \%\end{array}$ & $57(69.5 \%)$ & $53(75.7 \%)$ & 0.394 & $13(15.9 \%)$ & $13(18.6 \%)$ & 0.657 \\
\hline Lipid lowering drugs, $\%$ & $69(84.1 \%)$ & $60(85.7 \%)$ & 0.788 & $32(39.0 \%)$ & $30(42.9 \%)$ & 0.632 \\
\hline Antihypertensive drugs, $\%$ & $3(3.7 \%)$ & $6(8.6 \%)$ & 0.201 & $3(3.7 \%)$ & $6(8.6 \%)$ & 0.201 \\
\hline Aspirin, $\%$ & $74(90.4 \%)$ & $64(91.4 \%)$ & 0.801 & $33(40.2 \%)$ & $31(44.3 \%)$ & 0.615 \\
\hline
\end{tabular}

vs. conventional group, ${ }^{\dagger} \mathrm{p}<0.01,{ }^{*} \mathrm{p}<0.05$

Table 3. Post-treatment Changes of Indices between the Two Groups in the Patients Not Receiving Statins and Antihypertensive Agents.

\begin{tabular}{|c|c|c|c|c|c|c|}
\hline & \multicolumn{2}{|c|}{ Post-treatment (mean $\pm \mathrm{SD})$} & \multirow[b]{2}{*}{$\mathrm{p}$ value } & \multicolumn{2}{|c|}{ Changing values } & \multirow[b]{2}{*}{$\mathrm{p}$ value } \\
\hline & $\begin{array}{l}\text { Allopurinol } \\
\text { group }(n=13)\end{array}$ & $\begin{array}{l}\text { conventional } \\
\text { group }(n=10)\end{array}$ & & $\begin{array}{l}\text { Allopurinol } \\
\text { group }(n=13)\end{array}$ & $\begin{array}{l}\text { conventional } \\
\text { group }(n=10)\end{array}$ & \\
\hline $\begin{array}{l}\text { Systolic blood pressure, } \\
\mathrm{mmHg}\end{array}$ & $121 \pm 5$ & $126 \pm 7$ & 0.104 & $2 \pm 4^{*}$ & $6 \pm 3$ & 0.016 \\
\hline $\begin{array}{l}\text { Diastolic blood pressure, } \\
\mathrm{mmHg}\end{array}$ & $73 \pm 6$ & $75 \pm 4$ & 0.435 & $2 \pm 3$ & $3 \pm 2$ & 0.306 \\
\hline HOMA-IR & $3.12 \pm 0.73$ & $3.32 \pm 0.64$ & 0.515 & $0.09 \pm 0.18$ & $0.20 \pm 0.12$ & 0.124 \\
\hline Serum uric acid, umol/L & $319 \pm 16^{\dagger}$ & $455 \pm 14$ & $<0.001$ & $-113 \pm 17^{\dagger}$ & $25 \pm 8$ & $<0.001$ \\
\hline hs-CRP, mg/L & $2.64 \pm 0.66$ & $2.73 \pm 0.49$ & 0.724 & $0.16 \pm 0.07^{\dagger}$ & $0.26 \pm 0.09$ & 0.009 \\
\hline carotid IMT, mm & $1.07 \pm 0.21$ & $1.08 \pm 0.18$ & 0.937 & $0.03 \pm 0.02^{*}$ & $0.05 \pm 0.02$ & 0.017 \\
\hline
\end{tabular}

not receiving statins and antihypertensive agents (Table 3).

\section{Comparison of the incidence of hypertension after treatment in the two groups}

A PP analysis showed that the allopurinol group had 3 patients with new-onset hypertension (3.7\%), which was less than in the conventional therapy group (6 cases, 8.6\%); however, this difference was not significant $\left(\chi^{2}=1.636, \mathrm{p}=\right.$ $0.201)$

All the lost cases in the allopurinol and conventional therapy groups were considered progression to hypertension. At the end of the test, telephone follow-ups showed that all excluded patients in the allopurinol group had not progressed to hypertension. However, among 16 excluded patients in the conventional therapy group, 4 patients had new-onset hypertension. Therefore, an ITT analysis showed that there were 6 patients with new-onset hypertension in the allopurinol group (6.8\%), which was also less than that in the conventional therapy group ( 12 cases, $13.6 \%, \chi^{2}=2.228, p=$ 0.136). These results indicated that the ITT analysis results were consistent with the PP analysis.

\section{Adverse reactions}

A total of 3 patients in the allopurinol group had vomiting during the treatment, which occurred at the beginning of the treatment when the drug dosage was gradually increased. Two cases had mild vomiting and the symptoms gradually relieved after the dose-increasing rate of the drug was 
slowed and the patients were allowed to continue and complete the test. One patient had severe vomiting and was withdrawn from the trial. One patient from the allopurinol group had mild diarrhea with mild symptoms lasting less than 2 days, thereby not affecting the test. Additionally, 3 patients from the allopurinol group had abnormal liver function, including 2 cases of increased aspartate aminotransferase and alanine aminotransferase levels (both $<100 \mathrm{U} / \mathrm{L}$; one patient was required to be withdrawn from the trial, and the other patient recovered after the use of liver protective drugs), and 1 patient had a significantly elevated transaminase and bilirubin levels and was excluded from the trial.

\section{Discussion}

Asymptomatic HUA is HUA without the onset of arthrolithiasis or kidney stones formed from uric acid. In addition to causing gouty arthritis (12), the risk of asymptomatic HUA is closely related to T2DM, hypertension, metabolic syndrome, cerebral stroke, and cardiovascular diseases (13-16). Recent studies have shown that the serum uric acid level is closely related to macrovascular complications in T2DM patients $(9,10)$. Therefore, the early and effective control of serum uric acid in T2DM patients with asymptomatic HUA may have great implications for reducing the risks of macrovascular diseases.

Previous studies have shown that the serum uric acid level is an important risk factor for the development of atherosclerosis. Kuo et al. (17) have shown that uric acid was associated with atherosclerosis and cardiac hypertrophy even after adjusting for factors, such as age, protein urine and hsCRP, in healthy subjects. In addition, Cicero et al. (18) found that the carotid IMT increased with an increase of the serum uric acid levels in the absence of risk factors in patient with coronary heart disease. HUA also plays an important role in the occurrence and development of atherosclerosis in T2DM patients. Li et al. (10) conducted a follow-up study in 1,026 T2DM patients in order to observe the relationship between uric acid and carotid atherosclerosis. It has been found that uric acid is positively correlated with the carotid IMT; after the correction of various factors (such as age, sex, and diabetes duration, etc.), the risk of patients with HUA for developing carotid artery plaque was 2.71fold higher than that of the patients with normal uric acid levels, which further indicated that the serum uric acid level is an independent risk factor for the occurrence and development of carotid atherosclerosis.

Previous research has shown that HUA may stimulate vascular smooth muscle cell proliferation and cause oxidative stress, thereby damaging vascular endothelial cells leading to endothelial dysfunction $(19,20)$. Meanwhile, the urate crystals may depose in the vascular wall of patients with HUA and promote the inflammation reaction, thereby elevating the levels of hs-CRP and promoting the development of atherosclerosis (21). de Carvalho Vidigal et al. (22) found that uric acid is positively related to hs-CRP after the adjustment for various factors, such as age, gender, and BMI; thus, uric acid may be able to predict the serum levels of hs-CRP. A recent randomized controlled study (23) showed that the patients with asymptomatic HUA had higher hs-CRP levels than the patients with normal uric acid, and allopurinol therapy could significantly lower the levels of hs-CRP. In the present study, after three years of conventional treatment, the levels of hs-CRP significantly increased in the patients with T2DM and asymptomatic HUA. In the allopurinol group, however, there was a substantial decline in the levels of hs-CRP. This further illustrates the correlation between the serum uric acid level and the vascular inflammation reaction. Active intervention on HUA can significantly reduce vascular inflammation and effectively prevent the progression of subclinical cardiovascular disease.

HUA and hypertension are closely associated. A recent cross-sectional study (24) showed that elevation of the serum uric acid level by $1 \mathrm{mg} / \mathrm{dL}$ corresponded to a 1.20 -fold increase in the risk of hypertension even after the correction of various factors (such as age, sex, BMI, and glomerular filtration rate). In a prospective cohort study, Takase et al. (25) followed up on 8,157 healthy individuals with medical checkups for an average period of 48.3 months. They found that the incidence of hypertension gradually increases with elevating baseline serum uric acid levels. HUA is an independent risk factor for the occurrence and development of hypertension. Presently, few randomized controlled studies have investigated the effect of controlling serum uric acid on blood pressure. Kanbay et al. (23) found that allopurinol therapy could improve the endothelial function in the patients with asymptomatic HUA and increase the glomerular filtration rate, thereby lowering the blood pressure level. Our findings show that allopurinol therapy reduced the systolic and diastolic blood pressure levels in the patients with T2DM and asymptomatic HUA by maintaining the serum uric acid concentration below $360 \mu \mathrm{mol} / \mathrm{L}$. Additionally, the incidence of new-onset hypertension in the allopurinol group showed a declining trend compared to that in the conventional treatment group. Because of the secondary outcome measures, further studies are needed with a larger sample size.

The effects of HUA on insulin resistance and blood lipids are also the pathophysiological basis of atherosclerosis. HUA may impact insulin resistance in two ways: 1) by reducing nitric oxide-mediated (NO-mediated) vasoconstriction, thereby impairing glucose absorption; and 2) by directly elevating the oxygen partial pressure, leading to a proinflammatory response and thus inducing insulin resistance (26). Moreover, serum uric acid and TG are closely related to each other (27), whereas a high TG level is an important residual cardiovascular risk. Even if the use of statins in patients with total cholesterol and low density lipoprotein-cholesterol have been strictly controlled, the risk of cardiovascular events in patients with TG levels $>2.26$ 
$\mathrm{mmol} / \mathrm{L}$ increased by $27 \%$ compared with the patients with TG levels $<2.26 \mathrm{mmol} / \mathrm{L}$ (28). In the present study, after the serum uric acid levels were effectively controlled by allopurinol therapy, both TG and HOMA-IR showed an increasing trend compared with the data before the treatment. However, comparison of therapy-related differences between the groups showed that allopurinol therapy was more effective at lowering the TG level than the conventional therapy group. Additionally, allopurinol therapy could reduce HOMA-IR and improve insulin resistance, further delaying the development of atherosclerosis in T2DM patients.

To date, very few randomized controlled studies have reported the effect of intervention therapy on the serum uric acid level in carotid atherosclerosis. Zhu et al. (29) have shown that programmed intervention hyperuricemia contributes to the improvement of metabolism and cardiovascular morbid change. However, this study excluded patients with T2DM. Previous reports have also shown that it takes approximately 3 to 5 years to observe visible changes in the carotid IMT in T2DM patients receiving multi factor intensive treatment $(30,31)$. However, according to the study by Higgins (32), obvious changes in the carotid IMT may be observed as early as one year after allopurinol treatment. Thus, we estimated the effects of allopurinol combined with multi factor intensive treatment on the progress of the carotid IMT after 3 years. In the present study, a stringent low-purine diet and aggressive treatment were used to maintain the blood pressure, blood lipid, and blood glucose levels. However, during the 3-year follow-up period, the patients in the conventional therapy group had gradually increased uric acid levels due to kidney injury caused by HUA, with an obviously increasing trend in the carotid IMT, although the patients in the allopurinol group did not have significantly lower carotid IMT levels compared with the conventional therapy group, even after strict control of the serum uric acid levels. A comparison of therapy-related differences between the groups showed that allopurinol therapy was more effective at reducing the carotid IMT than conventional therapy. Further analysis of the findings found that allopurinol treatment was more effective in reducing the carotid IMT than conventional treatment in the patients not receiving statins and antihypertensive agents, thereby suggesting that allopurinol delays the progress of atherosclerosis.

A previous study has also found that allopurinol can significantly reduce the carotid IMT progression after 1 year of treatment compared with a placebo in patients with recent ischemic stroke and transient ischemic attack (32). However, our trial differed from that study in several ways. First, we included patients with T2DH and asymptomatic HUA, while the previous trial included patients with recent ischemic stroke and transient ischemic attack. Second, the previous study was performed in a Caucasian population, while our study was performed in an Asian population. Furthermore, our trial is the first to identify the effects of allopurinol on the carotid IMT in Asians. Third, the findings in our trial indicated that the effective control of serum uric acid levels by allopurinol can decrease insulin resistance and serum TG levels, which was not found in the previous trial. Finally, our study had a larger sample size and longer follow-up time, which may lead to more credible results.

It should be pointed out that the beneficial effects of allopurinol therapy observed in this study may be the consequence of both lowering the blood uric acid levels and inhibiting the xanthine oxidase (XO) enzyme system. Some studies have suggested that the benefit of blocking the XO system on endothelial dysfunction and cardiovascular disease may be related to the inhibition of XO-associated oxidative stress as opposed to the lowering of uric acid. However, Ogino et al. (33) found that benzbromarone, which is not a XO inhibitor, could improve the inflammatory markers and insulin resistance in patients with congestive heart failure, suggesting a direct effect of lowering the uric acid levels on inflammation.

There are several limitations associated with this study. First, our study was limited by the open-label design and the lack of a placebo control. Second, allopurinol may cause severe allergic reactions. Although this side effect did not occur during the study, the detection of HLA-5801 may reduce the occurrence of severe allergic reactions caused by allopurinol. Third, we did not have access to the personal lifestyle of each patient, such as vegetable and fruit consumption histories. The lack of such information may potentially lead to bias in the effect of allopurinol. Furthermore, we did not detect the pulse wave velocity (PWV) and flowmediated endothelium-dependent vasodilatation function (FMD), which are good indices for the evaluation of early atherosclerosis. Therefore, the results of this study must be confirmed by additional prospective studies with larger population sizes and longer follow-up periods to investigate whether such a strategy can provide durable benefits.

\section{Conclusion}

This study demonstrated that positive and effective control of the serum uric acid level may improve insulin resistance, decrease the serum levels of TG, hs-CRP, control blood pressure, and reduce the carotid IMT, thereby delaying the development of atherosclerosis in patients with $\mathrm{T} 2 \mathrm{DH}$ and asymptomatic HUA.

\section{The authors state that they have no Conflict of Interest (COI).}

\section{References}

1. Cardiovascular Department of Chinese Medical Doctor Association E. The Chinese expert consensus recommendations for diagnosis and treatment of asymptomatic hyperuricemia complicated with cardiovascular diseases. China Medical Herald 11: 19951999, 2009 (in Chinese).

2. Miao Z, Li C, Chen Y, et al. Dietary and lifestyle changes associated with high prevalence of hyperuricemia and gout in the Shandong coastal cities of Eastern China. J Rheumatol 35: 1859-1864, 2008 . 
3. Zhu Y, Pandya BJ, Choi HK. Comorbidities of gout and hyperuricemia in the US general population: NHANES 2007-2008. Am J Med 125: 679-687.e1, 2012.

4. Lv Q, Meng XF, He FF, et al. High serum uric acid and increased risk of type 2 diabetes: a systemic review and meta-analysis of prospective cohort studies. PLoS One 8: e56864, 2013.

5. Sluijs I, Beulens JW, van der AD, Spijkerman AM, Schulze MB, van der Schouw YT. Plasma uric acid is associated with increased risk of type 2 diabetes independent of diet and metabolic risk factors. J Nutr 143: 80-85, 2013.

6. Gaede P, Lund-Andersen H, Parving HH, Pedersen O. Effect of a multifactorial intervention on mortality in type 2 diabetes. $\mathrm{N}$ Engl J Med 358: 580-591, 2008.

7. Dahlof B, Devereux RB, Kjeldsen SE, et al. Cardiovascular morbidity and mortality in the Losartan Intervention For Endpoint reduction in hypertension study (LIFE): a randomised trial against atenolol. Lancet 359: 995-1003, 2002.

8. Szwejkowski BR, Gandy SJ, Rekhraj S, et al. Allopurinol reduces left ventricular mass in patients with type 2 diabetes and left ventricular hypertrophy. J Am Coll Cardiol 62: 2284-2293, 2013.

9. Fukui M, Tanaka M, Shiraishi E, et al. Serum uric acid is associated with microalbuminuria and subclinical atherosclerosis in men with type 2 diabetes mellitus. Metabolism 57: 625-629, 2008.

10. Li Q, Yang Z, Lu B, et al. Serum uric acid level and its association with metabolic syndrome and carotid atherosclerosis in patients with type 2 diabetes. Cardiovasc Diabetol 10: 72, 2011.

11. Levey AS, Stevens LA, Schmid CH, et al. A new equation to estimate glomerular filtration rate. Ann Intern Med 150: 604-612, 2009.

12. Wang Y, Yan S, Li C, et al. Risk factors for gout developed from hyperuricemia in China: a five-year prospective cohort study. Rheumatol Int 33: 705-710, 2013.

13. Goncalves JP, Oliveira A, Severo M, Santos AC, Lopes C. Crosssectional and longitudinal associations between serum uric acid and metabolic syndrome. Endocrine 41: 450-457, 2012.

14. Gur M, Sahin DY, Elbasan Z, et al. Uric acid and high sensitive C-reactive protein are associated with subclinical thoracic aortic atherosclerosis. J Cardiol 61: 144-148, 2013.

15. Kanbay M, Yilmaz MI, Sonmez A, et al. Serum uric acid independently predicts cardiovascular events in advanced nephropathy. Am J Nephrol 36: 324-331, 2012.

16. Li M, Hou W, Zhang X, Hu L, Tang Z. Hyperuricemia and risk of stroke: a systematic review and meta-analysis of prospective studies. Atherosclerosis 232: 265-270, 2014.

17. Kuo CF, Yu KH, Luo SF, et al. Role of uric acid in the link between arterial stiffness and cardiac hypertrophy: a cross-sectional study. Rheumatology 49: 1189-1196, 2010.

18. Cicero AF, Salvi P, D'Addato S, Rosticci M, Borghi C. Association between serum uric acid, hypertension, vascular stiffness and subclinical atherosclerosis: data from the Brisighella Heart Study. J Hypertens 32: 57-64, 2014.

19. Lanaspa MA, Sanchez-Lozada LG, Choi YJ, et al. Uric acid induces hepatic steatosis by generation of mitochondrial oxidative stress: potential role in fructose-dependent and -independent fatty liver. J Biol Chem 287: 40732-40744, 2012.

20. Yelken B, Caliskan Y, Gorgulu N, et al. Reduction of uric acid levels with allopurinol treatment improves endothelial function in patients with chronic kidney disease. Clin Nephrol 77: 275-282, 2012.

21. Kang DH, Park SK, Lee IK, Johnson RJ. Uric acid-induced Creactive protein expression: implication on cell proliferation and nitric oxide production of human vascular cells. J Am Soc Nephrol 16: 3553-3562, 2005

22. de Carvalho Vidigal F, de Lima Rosado LE, Paixao Rosado G, Lanes Ribeiro Rde C, Castro Franceschini Sdo C. Serum uric acid can predict higher C-reactive protein levels in apparently healthy men. Nutr Hosp 29: 935-940, 2014.

23. Kanbay M, Huddam B, Azak A, et al. A randomized study of allopurinol on endothelial function and estimated glomular filtration rate in asymptomatic hyperuricemic subjects with normal renal function. Clin J Am Soc Nephrol 6: 1887-1894, 2011.

24. Kuwabara M, Niwa K, Nishi Y, et al. Relationship between serum uric acid levels and hypertension among Japanese individuals not treated for hyperuricemia and hypertension. Hypertens Res 37: 785-789, 2014

25. Takase H, Kimura G, Dohi Y. Uric acid levels predict future blood pressure and new onset hypertension in the general Japanese population. J Hum Hypertens 28: 529-534, 2014.

26. Johnson RJ, Perez-Pozo SE, Sautin YY, et al. Hypothesis: could excessive fructose intake and uric acid cause type 2 diabetes? Endocr Rev 30: 96-116, 2009.

27. Conen D, Wietlisbach V, Bovet P, et al. Prevalence of hyperuricemia and relation of serum uric acid with cardiovascular risk factors in a developing country. BMC Public Health 4: 9, 2004.

28. Miller M, Cannon CP, Murphy SA, Qin J, Ray KK, Braunwald E. Impact of triglyceride levels beyond low-density lipoprotein cholesterol after acute coronary syndrome in the PROVE IT-TIMI 22 trial. J Am Coll Cardiol 51: 724-730, 2008.

29. Zhu WH, Fang LZ, Chen LY, Chen ZW, Dai HL, Chen JH. Follow-up study of programmed intervention of hyperuricemia in the prevention and treatment of cardiovascular morbid change. Natl Med J China 90: 662-666, 2010 (in Chinese).

30. Du JL, Liu JF, Men LL, et al. Effects of five-year intensive multifactorial intervention on the serum amyloid A and macroangiopathy in patients with short-duration type 2 diabetes mellitus. Chin Med J (Engl) 122: 2560-2566, 2009.

31. Kawasumi M, Tanaka Y, Uchino H, et al. Strict glycemic control ameliorates the increase of carotid IMT in patients with type 2 diabetes. Endocr J 53: 45-50, 2006.

32. Higgins $P$, Walters MR, Murray HM, et al. Allopurinol reduces brachial and central blood pressure, and carotid intima-media thickness progression after ischaemic stroke and transient ischaemic attack: a randomised controlled trial. Heart 100: 1085-1092, 2014.

33. Ogino K, Kato M, Furuse Y, et al. Uric acid-lowering treatment with benzbromarone in patients with heart failure: a double-blind placebo-controlled crossover preliminary study. Circ Heart Fail 3: $73-81,2010$

(C) 2015 The Japanese Society of Internal Medicine

http://www.naika.or.jp/imonline/index.html 